\title{
HÉRNIA VESICO-INGUINAL: RELATO DE CASO
}

\section{VESICO INGUINAL HERNIA: CASE REPORT}

Anna Maria Garcia Cardoso ${ }^{1}$; Luciana Borges Ferreira ${ }^{1}$; Letícia Debon ${ }^{1}$; Luiz Henrique Capaverde ${ }^{1}$; Maurício Krug Seabra ${ }^{1}$; Plínio Carlos Baú ${ }^{1}$.

\section{RESUMO}

Introdução: Hérnia é definida como uma protrusão anormal de um órgão ou tecido por um defeito em suas paredes circundantes. Embora uma hérnia possa ocorrer em vários locais do corpo, esses defeitos mais comumente envolvem a parede abdominal. Hérnias na região inguinal geralmente contém o omento e o intestino delgado. Entretanto, raramente pode conter conteúdos incomuns, como apêndice, ovário, bexiga urinária, cólon sigmóide e ceco. A correção cirúrgica das hérnias abdominais está entre os três procedimentos mais realizados por cirurgiões nos Estados Unidos. Relato do caso: Paciente masculino, 49 anos, sobrepeso, previamente hígido, encaminhado ambulatorialmente por hérnia inguinal bilateral, sintomático à direita. Há cerca de 5 anos observou aparecimento de hérnia inguinal à direita, tendo dor desde então, com piora no último ano, quando refere ter iniciado também com diminuição de jato miccional. Tomografia Computadorizada evidenciou hérnias inguinais, mais volumosa à direita, observando-se neste lado herniação da bexiga urinária. Internou eletivamente para realizar herniorrafia inguino-escrotal à direita, tendo sido optada por técnica de Lichtenstein. Procedimento sem intercorrências. Evolução adequada no pós operatório, apresentando na revisão boa cicatrização de ferida operatória, melhora da dor e dos sintomas urinários. Discussão: A incidência de bexiga fazendo parte de uma hérnia inguinal, chamada por Lavine, em 1951, de "cistocele escrotal", é de 1-4\%, ocorrendo com mais frequência em indivíduos com mais de 50 anos de idade com história de correção de hérnia inguinal. Em função da raridade desse diagnóstico, há uma certa dificuldade de encontrar dados recentes sobre esse tema.

Palavras-chave: Hernia. Hérnia Inguino-Vesical. Bexiga.

\begin{abstract}
Introduction: Hernia is defined as an abnormal protrusion of an organ or tissue from a defect in its surrounding walls. Although a hernia can occur in multiple locations on the body, these defects most commonly involve the abdominal wall. Hernias in the groin usually contain the omentum and small intestine. However, it may rarely contain unusual contents such as appendix, ovary, urinary bladder, sigmoid colon and cecum. Surgical correction of abdominal hernias is among the three procedures most commonly performed by surgeons in the US. Case report: Male patient, $49 \mathrm{y}$, overweight, previously healthy, referred to the clinic for bilateral inguinal hernia, symptomatic on the right. About 5 years ago, he observed the appearance of a right inguinal hernia, with pain since then, worsening in the last year, when he reports that it also started with a decrease in the voiding stream. Computed tomography showed inguinal hernias, more voluminous on the right, with urinary bladder herniation being observed on this side. Electively hospitalized to perform inguinal-scrotal herniorrhaphy on the right, the Lichtenstein technique was chosen. Procedure without complications. Adequate postoperative evolution, showing good surgical wound healing in the review, improvement in pain and urinary symptoms. Discussion: The incidence of bladder as part of an inguinal hernia, called "scrotal cystocele" by Lavine in 1951, is 1-4\%, occurring more frequently in individuals over 50 years of age with a history of hernia repair inguinal. Due to the rarity of this diagnosis, there is some difficulty in finding recent data on this topic.
\end{abstract}

Keywords: Hernia. Inguinoscrotal Bladder Hernias. Bladder.

\section{INTRODUÇÃO}

Uma hérnia é definida como uma protrusão anormal de um órgão ou tecido por um defeito em suas paredes circundantes. Embora uma hérnia possa ocorrer em vários locais do corpo, esses defeitos mais comumente envolvem a parede abdominal. As hérnias inguinais são mais frequentes na prática clínica e podem ser divididas em diretas ou indiretas, dependendo se estiverem medial ou lateralmente em relação aos vasos epigástricos inferiores respectivamente. As hérnias diretas são causadas por um enfraquecimento da musculatura da parede posterior do canal inguinal e ocorrem no triângulo de Hasselbach, segmento mais frágil da fáscia transversalis. As hérnias

${ }^{1}$ Hospital São Lucas, Pontifícia Universidade Católica do Rio Grande do Sul, Serviço de Cirurgia Geral e Cirurgia do Aparelho Digestivo - Porto Alegre - RS - Brasil 
indiretas são secundárias a alterações congênitas decorrentes do não fechamento do conduto peritoneovaginal ${ }^{1}$. As queixas mais frequentes de pacientes com hérnia inguinal são sensação de peso ou dor mal definida associada aos esforços, acompanhada ou não de abaulamento na região ${ }^{2}$. Já as hérnias femorais correspondem a menos de $5 \%$ das hérnias da região da virilha. São mais comuns em mulheres obesas acima de 45 anos. Nessas hérnias, o saco herniário se anuncia através do canal femoral, medialmente aos vasos femorais ${ }^{3}$. A classificação da European Hernia Society (EHS) leva em consideração o tamanho e localização anatômica $(\mathrm{L}=$ lateral, $\mathrm{M}=$ medial, $\mathrm{F}=$ femoral), além de as letras $\mathrm{P}$ e $\mathrm{R}$ poderem demonstrar, respectivamente, uma hérnia primária ou recorrente ${ }^{4}$.

A correção cirúrgica das hérnias abdominais está entre os três procedimentos mais realizados por cirurgiões nos Estados Unidos, totalizando 700.000 operações por ano; esses números demonstram a alta prevalência dessa condição, que acomete todas as faixas etárias, em ambos os sexos ${ }^{1}$. Hérnias na região inguinal geralmente contém o omento e o intestino delgado. Entretanto, raramente pode conter conteúdos incomuns, como apêndice, ovário, bexiga urinária, cólon sigmóide e ceco ${ }^{5-11}$.

Tem-se como objetivo apresentar o relato de caso de paciente com hérnia inguino-vesical, operado no hospital do serviço dos autores. Este trabalho foi aprovado pelo Comitê de Ética e Pesquisa da mesma instituição, sob o número 4.839.957.

\section{RELATO DO CASO}

$\begin{array}{ll}\text { Paciente } & \begin{array}{l}\text { masculino, } 49 \text { anos, } \\ \text { previamente } \\ \text { hígido, } \\ \text { encaminhado } \\ \text { ambulatorialmente por }\end{array}\end{array}$
hérnia inguinal bilateral, sintomático à direita. Há cerca de 5 anos observou aparecimento de hérnia inguinal à direita, tendo dor desde então, com piora no último ano, quando refere ter iniciado também com diminuição de jato miccional. Inicialmente conseguia realizar redução espontânea, sendo que nos últimos 3 meses houve encarceramento por duas vezes, em ambas tendo sido possivel a redução manual em ambiente hospitalar e em uma delas sendo necessária sondagem vesical de alivio, sem necessidade de intervenção cirúrgica. Ao exame físico, apresentava hérnia inguinoescrotal média à direita, palpado defeito importante do canal inguinal, com redução à manobra sem dificuldade; à esquerda defeito não palpável. Ultrassom das regiões inguinais evidenciou herniação de conteúdo intraabdominal nas regiões inguinais, mais pronunciada à direita, sem sinal de encarceramento ou de inflamação aguda. $\mathrm{Na}$ última consulta em emergência por encarceramento, o paciente apresentava dor intensa periumbilical, com irradiação para a região inguinal direita, realizando Tomografia Computadorizada antes da redução, evidenciando hérnias inguinais, mais volumosa à direita, observando-se neste lado herniação da bexiga urinária (Figura 1).

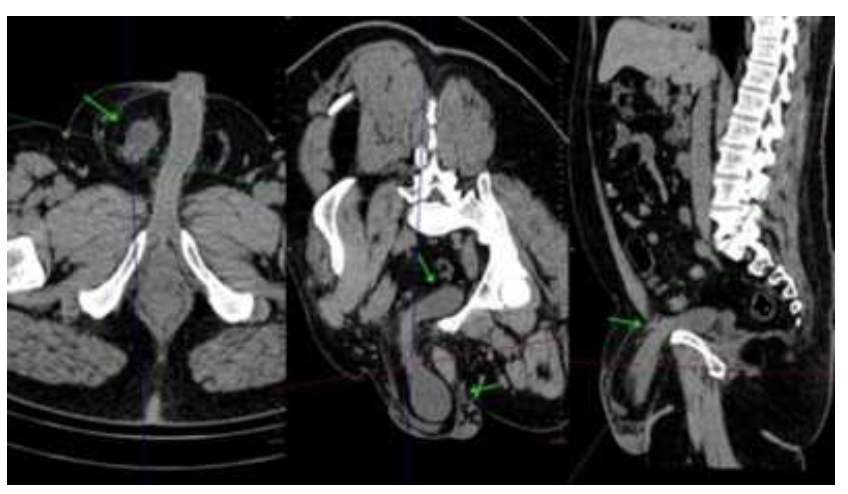

Figura 1. Cortes Axial; Coronal obliquo; Sagital com flechas apontando para bexiga.

Neste lado observa-se também pequena quantidade de líquido livre. Internou eletivamente para realizar herniorrafia inguino-escrotal à direita, tendo sido optada por técnica de Lichtenstein, identificando-se o anel herniário com o defeito da parede posterior com insinuação da bexiga, dissecção e redução do mesmo e fixação de tela, revisão hemostática e fechamento por planos. Procedimento sem intercorrências. Evolução adequada, tendo alta no primeiro dia pós-operatório, apresentando na revisão boa cicatrização de ferida operatória, melhora da dor e dos sintomas urinários.

\section{DISCUSSÃO}

A incidência de bexiga fazendo parte de uma hérnia inguinal, chamada por Lavine, em 1951, de "cistocele escrotal"12, é de $1-4 \%$, ocorrendo com mais frequência 
em indivíduos com mais de 50 anos de idade com história de correção de hérnia inguinal. Em função da raridade desse diagnóstico, há uma certa dificuldade de encontrar dados recentes sobre esse tema.

A fisiopatologia das hérnias inguinais de bexiga pode estar relacionada à tração da bexiga juntamente com uma bainha de peritônio que forma seu saco, por meio de um ponto fraco na fáscia abdominal ${ }^{13}$. Alguns fatores, incluindo obstrução da saída da bexiga, obesidade, diminuição do tônus vesical e fraqueza da musculatura pélvica, estão associados ao seu desenvolvimento.

Alguns estudos apontam que as hérnias da bexiga são mais comuns no lado direito ${ }^{14-16}$. Os pacientes são geralmente assintomáticos, mas podem apresentar sintomas inespecíficos, incluindo aumento da frequência urinária, urgência, noctúria e hematúria ${ }^{15}$. Os estudos de imagem podem ser realizados quando há suspeita de hérnia da bexiga, incluindo tomografia computadorizada, ultrassom e cistografia.

Menos de $7 \%$ das hérnias da bexiga são diagnosticadas no pré-operatório, 16\% são diagnosticadas no pós-operatório por causa de complicações ${ }^{16}$. O tratamento padrão para hérnias inguinais envolvendo a bexiga é o reparo cirúrgico (herniorrafia). O risco de lesão da bexiga durante a herniorrafia foi relatado como $12 \% 17$. Portanto, o diagnóstico preciso de hérnias inguinoescrotais da bexiga é importante para evitar lesões na bexiga durante 0 reparo cirúrgico ${ }^{18}$.

\section{REFERÊNCIAS}

1. Burcharth, J. The epidemiology and risk factors for recurrence after inguinal hernia surgery. Dan Med J. 2014;61(5):B4846.

2. Mitura K, Śmietański M, Kozieł S, Garnysz K, Michałek I. Factors influencing inguinal hernia symptoms and preoperative evaluation of symptoms by patients: results of a prospective study including 1647 patients. Hernia. 2018;22(4):585-91.

doi: $10.1007 / \mathrm{s} 10029-018-1774-4$.

3. Goethals A, Azmat CE, Adams CT. Femoral Hernia. 2020 Nov 4. In: StatPearls [Internet]. Treasure Island (FL): StatPearls Publishing; 2020.
4. Miserez M, Alexandre J, Campanelli G, Corcione F, Cuccurullo D, Pascual $\mathrm{M}$, et al. The European hernia society groin hernia classication: simple and easy to remember. Hernia. 2007;11(2):113-6.

doi: $10.1007 / \mathrm{s} 10029-007-0198-3$.

5. Patoulias D, Kalogirou M, Patoulias I. Amyand's Hernia: an Up-to-Date Review of the Literature. Acta Medica (Hradec Kralove). 2017;60(3):131-4. doi: 10.14712/18059694.2018.7.

6. Guenther T, Theodorou C, Grace N, Rinderknecht T, Wiedeman J. De Garengeot hernia: a systematic review. Surg Endosc. 2021;35(2):503-13. doi: $10.1007 / \mathrm{s} 00464-020-07934-5$.

7. Regelsberger-Alvarez CM, Pfeifer C. Richter Hernia. 2020 Mar 29. In: StatPearls [Internet]. Treasure Island (FL): StatPearls Publishing; 2020.

8. Pinto J, Viana C, Pereira A, Falcão J. Littré's hernia. BMJ Case Rep. 2019;12(2):bcr-2018-228784.

doi: $10.1136 /$ bcr-2018-228784.

9. Alves A Jr, Maximiano L, Fujimura I, Pires PW, Birolini D. Grynfelt hernia. Arq Gastroenterol. 1996;33(1):32-5.

10. Perrakis A, Velimezis G, Kapogiannatos G, Koronakis D, Perrakis E. Spigel hernia: a single center experience in a rare hernia entity. Hernia. 2012;16(4):439-44. doi: $10.1007 / \mathrm{s} 10029-012-0925-2$.

11. Goyal S, Shrivastva M, Verma R, Goyal S. "Uncommon Contents of Inguinal Hernial Sac": A Surgical Dilemma. Indian J Surg. 2015;77(Suppl 2):305-9. doi: $10.1007 / \mathrm{s} 12262-013-0806-7$..

12. Levine B. Cistocele escrotal. JAMA. 1951;147(15):1439-41. doi:

10.1001/jama.1951.73670320003013a.

13. Elkbuli A, Narvel R, McKenney $M$, Boneva D. Inguinal bladder hernia: A case report and literature review. Int $\mathrm{J}$ Surg Case Rep. 2019;58:208-11. doi: 10.1016/j.ijscr.2019.04.040.

14. Bisharat M, O'Donnell M, Thompson $\mathrm{T}$, MacKenzie N, Kirkpatrick D, Spence $\mathrm{R}$, et al. Complications of inguinoscrotal bladder hernias: a case series. Hernia. 2009;13(1):81-4. doi: 10.1007/s10029-008-0389-6.

15. Ugur M, Atci N, Oruc C, Akkucuk S, Aydogan A. Left inguinal bladder hernia that causes dilatation in the ureter. Arch Iran Med. 2016;19(5):376-8. 
16. Watson L. Hernia Anatomy, Etiology, Symptoms Diagnosis, Differential Diagnosis, Prognosis and Treatment. 3rd ed. St. Louis (MO): CV Mosby, 1948.

17. Catalano O, 1997. US evaluation of inguinoscrotal bladder hernias: Report of three cases. Clin Imaging. 1997;21(2):126-8.

doi: 10.1016/s0899-7071(96)00018-6.

18. Gomella L. The Surgical Implications of Herniation of the Urinary Bladder. Arch Surg. 1985:120(8):964-7. doi: 10.1001/archsurg. 1985.01390320084 018.
Fonte de financiamento: Não

Conflito de interesses: Não

Data de Submissão: 05 Dezembro 2021

Decisão final: 30 Agosto 2021

\section{Autor de Correspondência:}

Anna Maria Garcia Cardoso

E-mail: annamariagarciacardoso@gmail.com 